\title{
Combination Chemotherapy with Cisplatin and VM-26 in Advanced Transitional Cell Carcinoma of the Bladder*
}

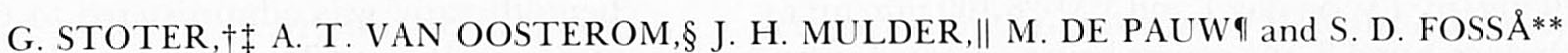

On behalf of the EORTC Genito-Urinary Group $\dagger \dagger$

$\dagger$ Department of Oncology, Free University Hospital, Amsterdam, The Netherlands, §Department of Clinical Oncology, University Hospital, Leiden, The Netherlands, ||Department of Internal Medicine, Radiotherapy Institute, Rotterdam, The Netherlands, 9 EORTC Data Center, Institut Jules Bordet, Bruxelles, Belgium and **Norwegian Radium Hospital, Oslo, Norway

\begin{abstract}
Forty-one evaluable patients with bidimensionally measurable metastases of transitional cell carcinoma of the bladder were treated with cisplatin $70 \mathrm{mg} / \mathrm{m}^{2}$ i.v. on day 1 and $V M-26100 \mathrm{mg} / \mathrm{m}^{2}$ i.v. on days 1 and 2, every 3 weeks. Response was evaluated after 2 treatment cycles. Complete response (CR) was achieved in 4 patients (10\%) and partial response $(P R)$ in 17 (41\%). The median response duration was 6 months. In this group of previously untreated patients the combination of cisplatin and VM-26 did not appear to yield better response rates than would be expected from cisplatin alone.
\end{abstract}

\section{INTRODUCTION}

PresentLy, cisplatin is the most active single agent in the treatment of advanced bladder cancer. Dosages of $70-120 \mathrm{mg} / \mathrm{m}^{2}$ i.v., every 3 weeks, or equivalents thereof, have yielded response rates of $30-40 \%$ in pretreated patients and $40-50 \%$ in nonpretreated subjects, with a median response duration of 6 months [1-5]. Various combinations of cisplatin with agents such as adriamycin, cyclophosphamide and 5 -fluorouracil have failed to improve these results [6-10].

In an attempt to improve the therapeutic results, the EORTC Urological Group initiated a phase II study (protocol 30802) with the combination of cisplatin and VM-26 based on the following rationale. First, a phase II study in the early 1970s conducted by this group with VM-26

Accepted 11 July 1983.

*This study was supported by NCI grant 2U10-CAl1488-13 and by a grant from Bristol-Myers Company, New York.

$\ddagger$ To whom requests for reprints should be addressed at: Free University Hospital, De Boelelaan 1117, 1007 MB Amsterdam, The Netherlands.

$\dagger \dagger$ Other participating investigators: T. Splinter, Rotterdam; L. Denis, Antwerp; J. P. Bergerat, Strassburg; G. Jakse, Innsbruck; B. Richards, York; M. Rozencweig, Brussels; C. Schulman, Brussels; and C. P. J. Vendrik, Utrecht. suggested that the drug may be effective in advanced bladder cancer. Two CRs and 3 PRs were observed in 30 heavily pretreated patients [11]. Second, Burchenal et al. have demonstrated synergism between platinum- and epipodophyllotoxin derivates [12]. Finally, the side-effects of the two drugs differ. Myelosuppression is the main adverse effect of VM-26, whereas cisplatin affects the bone marrow only mildly. The objectives of the study were to determine the response rate and duration of response of this combination.

\section{MATERIALS AND METHODS}

\section{Patients}

Patients were eligible for the study if they had histologically proven transitional cell cancer of the urinary tract with bidimensionally measurable distant metastases (lung, liver, lymph nodes, skin) of a pelvic tumour mass measurable by CT scan. Further requirements included a performance status (WHO scale $0-2$ ), creatinine clearance $\geqslant 40 \mathrm{ml} / \mathrm{min}$ and normal bone marrow function. Patients with previous treatment consisting of cisplatin or VM-26 were not accepted. Bone metastases, hepatomegaly and serous effusions were not accepted as measurable lesions, and patients with a history of congestive heart failure 
were excluded because of the vigorous hydration scheme.

Pretreatment evaluation included history and physical examination, a routine hematological and biochemical screen, creatinine clearance, IVP and chest X-ray. Except for IVP, these studies were repeated before each treatment cycle. CT-scan was performed prior to treatment and repeated after every second treatment cycle if necessary to measure indicator lesions.

The treatment regimen consisted of cisplatin $70 \mathrm{mg} / \mathrm{m}^{2}$ i.v. on day 1 and VM-26 $100 \mathrm{mg} / \mathrm{m}^{2}$ i.v. on days 1 and 2, the course being repeated every 3 weeks. Cisplatin was given over a 4-hr period with hydration. VM-26 was administered in $250 \mathrm{ml}$ of saline over $30 \mathrm{~min}$. For cases of severe bone marrow depression, dose reductions of $25-50 \%$ of VM-26 were required.

The definitions of response were according to the WHO criteria [13]. CR is defined as the disappearance of all known disease for at least 4 weeks and PR requires a $50 \%$ or more decrease in the product of the two largest perpendicular diameters of all measurable lesions for no less than 4 weeks, with no new lesions developing. Stable disease is considered as less than a $50 \%$ decrease or less than a $25 \%$ increase in measurable disease, with no new lesions developing, and progression is more than a $25 \%$ increase in measurable disease or the appearance of new lesions.

Patients were considered evaluable for response if they had completed a minimum of 2 cycles of therapy.

\section{Patient characteristics}

Fifty-eight patients were entered by 12 institutions. Eight patients were ineligible (Table l) for the following reasons: histology failed to show transitional cell carcinoma in 3 patients; performance status was $>2$ in another 3 patients; 1 patient had hepatomegaly only; and 1 had a second malignancy.

Nine patients were inevaluable for treatment response (Table 2). In 4 patients inadequate dosages were given, and 2 patients were treated with incorrect intervals. After l cycle of therapy, 1 patient refused further treatment, 1 patient developed renal failure due to obstructive uropathy and a third patient developed urosepsis without leukopenia.

The characteristics of the 41 evaluable patients are given in Table 3. The average age of patients was $61 \mathrm{yr}$ and males dominated the subject population $6: 1$. Thirteen patients underwent cystectomy with ileal conduit and 18 patients received radiotherapy to the bladder. Topical chemotherapy was administered to 6 patients: 5 intravesically and 1 intra-arterially. Six patients had tumour limited to the pelvis, 2 of which had received prior radiotherapy.

No patient received previous systemic chemotherapy.

Table 1. Reasons for ineligibility

\begin{tabular}{ll}
\hline No transitional cell cancer & 3 patients \\
Performance status too low & 3 \\
Unmeasurable indicator lesion & 1 \\
Second malignancy & 1 \\
Total ineligible & $8 / 58$ \\
\hline
\end{tabular}

Table 2. Reasons for inevaluability

\begin{tabular}{ll} 
Inadequate dosage & 4 patients \\
Inadequate treatment intervals & 2 \\
Treatment refused after l cycle & 1 \\
Renal failure after l cycle & 1 \\
Urosepsis & 1 \\
Total inevaluable & $9 / 58$ \\
\hline
\end{tabular}

Table 3. Characteristics of 41 evaluable patients

\begin{tabular}{ll}
\hline Sex ratio male: female & $6: 1$ \\
Average age & $61 \mathrm{yr}$ \\
Ileal conduit & 13 patients \\
Previous radiotherapy & 18 \\
Topical chemotherapy & 6 \\
Pelvic tumour only & 6 \\
\hline
\end{tabular}

Table 4. Results of treatment

\begin{tabular}{lcccc}
\hline \multicolumn{1}{c}{$\begin{array}{c}\text { Site of } \\
\text { metastases }\end{array}$} & $\begin{array}{c}\text { No. of } \\
\text { patients }\end{array}$ & CR $(\%)$ & PR $(\%)$ & Overall (\%) \\
\hline Lung & 14 & $2(14)$ & $6(43)$ & $8(57)$ \\
Lymph nodes & 17 & $2(12)$ & $7(41)$ & $9(53)$ \\
Liver & 4 & - & $2(50)$ & $2(50)$ \\
Pelvis & 6 & - & $2(33)$ & $2(33)$ \\
Total & 41 & $4(10)$ & $17(41)$ & $21(51)$ \\
\hline
\end{tabular}




\section{RESULTS}

Forty-one evaluable patients were treated with an average number of 4 cycles (range 2-9). Four achieved CR (10\%) and 17 PR (41\%), for an overall response rate of $51 \%$. Eleven patients had stable disease, 7 progressed and 2 died secondary to their malignancy. Response was evaluated according to the site of metastases (Table 4). Of the 6 patients in whom the tumor was confined to the pelvis, 2 received prior radiotherapy: 1 had stable disease and the other had progression of disease after 2 cycles. In the 4 non-irradiated cases, 2 achieved PR, 1 had stable disease and 1 had progression.

The median duration of response was 6 months. The median survival of patients with CR was 12 months. Patients with PR and no change had a median survival of 6 months and patients with progressive disease had a median survival of only 3 months.

The major toxity was nausea and vomiting, which led to cessation of treatment in 6 patients. Leukocytopenia $\left(1400-3800 / \mathrm{mm}^{3}\right.$ ) was observed in 10 patients and required dose reductions in 7 . Thrombocytopenia $\left(<100,000 / \mathrm{mm}^{3}\right)$ was observed in one patient $\left(85,000 / \mathrm{mm}^{3}\right)$. No sepsis or bleeding complications occurred.

\section{DISCUSSION}

The overall response rate of $51 \% \quad(95 \%$ confidence limits: 36-67\%) with a median response duration of 6 months in this patient population without prior chemotherapy does not differ from cisplatin as a single agent. Therefore, this study fails to confirm a synergistic effect between cisplatin and the epipodophyllotoxin derivate, and it appears that VM-26 has only weak activity in advanced bladder cancer. VM-26, when used as a single agent at a dose of $20-30 \mathrm{mg} / \mathrm{m}^{2}$ i.v. daily $\times 5$, every 3 weeks, has recently been reported as ineffective, although the study can be criticized for suboptimal doses and extensively pretreated patients [14].

The results of this study support that cisplatin combination chemotherapy is not proven superior to single-agent cisplatin in bladder cancer [5-10], and since multidrug treatment may also increase toxicity, combination chemotherapy with cisplatin is not recommended for routine treatment of transitional cell cancer of the bladder.

\section{REFERENCES}

1. HERR HW. Cis-diamminedichloroplatinum II in the treatment of advanced bladder cancer. J Urol 1980, 123, 853-855.

2. Ostrow S, Egorin MJ, Hahn D et al. Cis-dichlorodiammine-platinum and adriamycin therapy for advanced gynaecological and genitourinary neoplasms. Cancer, 1980, 46, 1715-1721.

3. PETERS PC, O'NeILl MR. Cis-diamminedichloroplatinum as a therapeutic agent in metastatic transitional cell carcinoma. J Urol 1980, 123, 375-377.

4. YAGODA A. Chemotherapy of metastatic bladder cancer. Cancer 1980, 45, 1879-1888

5. Stoter G, Williams SD, Einhorn LH. Genitourinary tumors. In: Pinedo HM, ed. Cancer Chemotherapy Annual 3. Amsterdam, Excerpta Medica, 1981, 322-325.

6. YAGODA A, WATSON RC, WhitMore WF. Cisplatinum (DDP) regimens in bladder cancer. Proc Am Soc Clin Oncol 1979, 20, 347.

7. Troner MB. Cyclophosphamide, adriamycin and platinum (CAP) in the treatment of urothelial malignancy. Proc Am Assoc Cancer Res 1979, 20, 117.

8. SAMUel ML. CISCA combination chemotherapy. In: JohnSOn DE, SAMUELS ML, eds Cancer of the Genito-urinary Tract. New York, Raven Press, 1979, 97-106.

9. Williams SD, Einhorn LH, DONOHUE JP. Cisplatin combination chemotherapy of bladder cancer. Cancer Clin Trials 1979, 2, 335-338.

10. Mulder JH, Foss $\AA$ SD, de Pauw M, van Oosterom AT. Cyclophosphamide, adriamycin and cisplatin combination chemotherapy in advanced bladder carcinoma: an EORTC phase II study. Eur J Cancer Clin Oncol 1982, 18, 111-112.

11. Pavone-Macaluso M and the EORTC Genito-Urinary Cooperative Group Single drug chemotherapy of bladder cancer with adriamycin, VM-26 or bleomycin. Eur Urol 1976, 2, 138-141.

12. Burchenal JH, Kalaher K, Lokys L, Gale G. Studies of cross-resistance, synergistic combinations and blocking of acvitity of platinum derivatives. Biochimie 1978, 60, 961-965.

13. WHO Handbook for Reporting Results of Cancer Treatment. WHO, Geneva, 1979, Offset Publication 48, 23-24.

14. QAZi R, Elson P, Khandekar JD. Phase II evaluation of VM-26 in patients with metastatic transitional cell carcinoma of the urinary tract: an Eastern Cooperative Oncology Group Study. Cancer Treat Rep 1982, 66, 405-406. 